\title{
Arbitrary groups as two-point stabilisers of symmetric groups acting on partitions
}

\author{
J. P. James
}

Received: 16 February 2005 / Accepted: 9 February 2006 /

Published online: 22 August 2006

(C) Springer Science + Business Media, LLC 2006

\begin{abstract}
We give a short, direct proof that given any finite group $G$ there exist positive integers $k$ and $l$ and partitions $\alpha_{1}$ and $\alpha_{2}$ of $\{1, \ldots, k l\}$ into $l$ subsets of size $k$ such that $\left(S_{k l}\right)_{\alpha_{1}, \alpha_{2}} \cong G$.

The method used will also show that given any finite group $G$ there exists a regular bipartite graph whose automorphism group is isomorphic to $G$.
\end{abstract}

Keywords Symmetric groups acting on partitions · Regular bipartite graphs ·

Two-point stabilisers

\section{Introduction}

Let $k$ and $l$ be positive integers such that $k, l \geq 2$ and let $\Omega_{k, l}$ be the set of all partitions of $\{1, \ldots, k l\}$ into $l$ distinct subsets of size $k$. In a previous paper [1] the author showed that there exist $\alpha_{1}, \alpha_{2} \in \Omega_{k, l}$ such that $\left(S_{k l}\right)_{\alpha_{1}, \alpha_{2}}=1$ if and only if $k \geq 3$ and $l \geq \max \{8, k+3\}$. In light of this result it is natural to ask which finite groups can occur as two-point stabilisers of a symmetric group acting on partitions. This is answered by the following theorem.

Theorem 1.1. Let $G$ be a finite group. There exist positive integers $k, l$ and partitions $\alpha_{1}, \alpha_{2} \in \Omega_{k, l}$ such that $\left(S_{k l}\right)_{\alpha_{1}, \alpha_{2}} \cong G$.

In fact Theorem 1.1 is a corollary of a result of Kantor [3] who proved that given a finite group $G$ there exists a symmetric design with automorphism group isomorphic to $G$. For a given $G$, the proof gives values of $k$ and $l$ which are both exponential in

J. P. James $(\bowtie)$

Department for Pure Mathematics and Mathematical Statistics, Centre for Mathematical Sciences, University of Cambridge, Wilberforce Road, Cambridge, CB3 0WB, UK

e-mail: J.P.James@dpmms.cam.ac.uk 
$|G|^{2}$. The aim of this paper is to give a simple direct proof of Theorem $1.1 \mathrm{in}$ which far smaller values of $k$ and $l$ are necessary. We will prove the following result

Theorem 1.2. Let $G$ be a non-trivial finite group. Set $k=4$ and $l=2(r+1)|G|$ where $r$ is the minimum number of elements needed to generate $G$. There exist $\alpha_{1}$, $\alpha_{2} \in \Omega_{k, l}$ such that $\left(S_{k l}\right)_{\alpha_{1}, \alpha_{2}} \cong G$.

\subsection{Fences}

In [1] the author defined fences. We recall the definition (in a slightly more general form) and the most important result.

Definition 1.3. Let $\Omega$ be a finite set and let $k$ be a positive integer such that $2 \leq k<|\Omega|$. $\mathrm{A}(k, \Omega)$-fence is a collection $\Gamma$ of $|\Omega|$ distinct $k$-subsets of $\Omega$ such that each member of $\Omega$ occurs in precisely $k$ members of $\Gamma$.

Remark 1.4. In design theory terminology a $(k, \Omega)$-fence is the blocks of a $1-(|\Omega|, k, k)$ design.

Lemma 1.5. Let $\Omega$ be a finite set and set $l=|\Omega|$. Let $k$ be a positive integer such that $2 \leq k<$ l and $\Gamma$ be a $(k, \Omega)$-fence. There exist partitions $\alpha_{\Gamma}, \beta_{\Gamma} \in \Omega_{k, l}$ such that $\left(S_{k l}\right)_{\alpha_{\Gamma}, \beta_{\Gamma}} \cong \operatorname{Sym}(\Omega)_{\Gamma}$.

Proof: See Lemma 2.3 and Lemma 3.3 in [1].

So to prove Theorem 1.2 it is enough, given $G$ which can be generated by $r$ elements, to construct a $(4, \Omega)$-fence $\Gamma_{G}$, where $\Omega$ is a set of size $2(r+1)|G|$, with stabiliser isomorphic to $G$.

Remark 1.6. The proof of Lemma 1.5 in [1] shows that given a $(k, \Omega)$-fence we can construct a $k$-regular bipartite graph $\mathcal{G}$ such that $\operatorname{Aut}_{\mathrm{b}}(\mathcal{G}) \cong \operatorname{Sym}(\Omega)_{\Gamma}$ where $\operatorname{Aut}_{\mathrm{b}}(\mathcal{G})$ is the group of automorphisms of $\mathcal{G}$ fixing the bipartite blocks of $\mathcal{G}$. Some more work shows that there exists a regular bipartite graph such that $\operatorname{Aut}(\mathcal{G}) \cong \operatorname{Sym}(\Omega)_{\Gamma}$. See [2] for details.

\section{Cyclic groups}

The Main Construction (detailed in Section 3) requires at least two distinct elements that generate the group. So we deal with cyclic groups separately.

2.1. The group of size two

Set $\Gamma_{C_{2}}=\Delta_{1} \cup \Delta_{2}$ where

$$
\begin{aligned}
& \Delta_{1}=\{\{1,2,3,4\},\{2,3,4,5\},\{3,4,5,6\},\{4,5,6,7\},\{5,6,7,8\}\} \\
& \Delta_{2}=\{\{6,2,8,1\},\{2,8,1,7\},\{8,1,7,3\}\} .
\end{aligned}
$$


We observe that $\Gamma_{C_{2}}$ is a $(4,\{1, \ldots, 8\})$-fence.

Lemma 2.1. The stabiliser of $\Gamma_{C_{2}}$ in $S_{8}$ has size two.

Proof: Consider a graph $\mathcal{K}_{C_{2}}$ with vertex set $\Gamma_{C_{2}}$ in which two sets are joined by an edge if they intersect in three points. Elements of $\left(S_{8}\right)_{\Gamma_{C_{2}}}$ act as automorphisms of $\mathcal{K}_{C_{2}}$. The connected components of $\mathcal{K}_{C_{2}}$ are a line of length five and a line of length three corresponding to $\Delta_{1}$ and $\Delta_{2}$. So if $g \in\left(S_{8}\right)_{\Gamma_{C_{2}}}$ then $g$ either fixes or reflects the line corresponding to $\Delta_{1}$ hence $g$ is either the identity or the involution $(1,8)(2,7)(3,6)(4,5)$.

\subsection{Other cyclic groups}

Let $n$ be an integer such that $n \geq 3$. Set $\Omega_{C_{n}}=\{0, \ldots, 4 n-1\}$ and $\Gamma_{C_{n}}=\bigcup_{i=0}^{n-1} \Delta_{i}$ where

$$
\begin{aligned}
\Delta_{i}= & \{\{4 i, \ldots, 4 i+3\},\{4 i+1, \ldots, 4 i+4\},\{4 i+2, \ldots, 4 i+5\}, \\
& \{4 i+3, \ldots, 4 i+10\}\}
\end{aligned}
$$

with addition modulo $4 n$. We observe that $\Gamma_{C_{n}}$ is a $\left(4, \Omega_{C_{n}}\right)$-fence.

Lemma 2.2. The stabiliser of $\Gamma_{C_{n}}$ in $\operatorname{Sym}\left(\Omega_{C_{n}}\right)$ is isomorphic to $C_{n}$.

Proof: As above $\operatorname{Sym}\left(\Omega_{C_{n}}\right)_{\Gamma_{C_{n}}} \leq \operatorname{Aut}\left(\mathcal{K}_{C_{n}}\right)$ where $\mathcal{K}_{C_{n}}$ is a graph with vertices $\Gamma_{C_{n}}$ in which two sets are joined if they intersect in three points. The connected components of $\mathcal{K}_{C_{n}}$ are $n$ lines of length four corresponding to the sets $\Delta_{0}, \ldots, \Delta_{n-1}$. So $g \in$ $\operatorname{Sym}\left(\Omega_{C_{n}}\right)_{\Gamma_{C_{n}}}$ permutes the sets $\Delta_{0}, \ldots, \Delta_{n-1}$.

We observe that the permutation $\sigma \in \operatorname{Sym}\left(\Omega_{C_{n}}\right)$ given by $i \sigma=i+4 \bmod 4 n$ stabilises $\Gamma_{C_{n}}$ and sends $\Delta_{j}$ to $\Delta_{j+1}$ where $j \in[0, n-1]$ and addition is modulo $n$. $\operatorname{So} \operatorname{Sym}\left(\Omega_{C_{n}}\right)_{\Gamma_{C_{n}}}$ is transitive on the connected components of $\mathcal{K}_{\Gamma_{C_{n}}}$ and contains a subgroup isomorphic to $C_{n}$. To show that $\operatorname{Sym}\left(\Omega_{C_{n}}\right)_{\Gamma_{C_{n}}} \cong C_{n}$ it is enough to show that the stabiliser of $\Delta_{0}$ in $\left(\operatorname{Sym}\left(\Omega_{C_{n}}\right)_{\Gamma_{C_{n}}}\right)$ is trivial.

Let $i \in[0, n-1]$ and suppose $g \in \operatorname{Sym}\left(\Omega_{C_{n}}\right)_{\Gamma_{C_{n}}}$ such that $\Delta_{i} g=\Delta_{i}$. So $g$ either fixes or reflects the corresponding connected component. Hence $g$ acts on $\operatorname{supp}\left(\Delta_{i}\right)=$ $\{4 i, \ldots, 4 i+5\} \cup\{4 i+10\}$ either as the identity or the involution $(4 i, 4 i+10)(4 i+$ $1,4 i+5)(4 i+2,4 i+4)$ where all addition is modulo $4 n$. We observe that

- the elements $4 i$ and $4 i+1$ are only contained in members of $\Delta_{i}$ and $\Delta_{i-1}$;

- the element $4 i+5$ is only contained in members of $\Delta_{i}$ and $\Delta_{i+1}$;

- the element $4 i+10$ is only contained in members of $\Delta_{i}$ and $\Delta_{i+2}$

where addition in indices is modulo $n$ and all other addition is modulo $4 n$. Hence $g$ can not act as an involution on $\operatorname{supp}\left(\Delta_{i}\right)$ otherwise it must send $\Delta_{i-1}$ to both $\Delta_{i+1}$ and $\Delta_{i+2}$ simultaneously. Therefore $g$ is the identity on $\operatorname{supp}\left(\Delta_{i}\right)$ and must stabilise $\Delta_{i+1}$. So the stabiliser of $\Delta_{0}$ in $\left(\operatorname{Sym}\left(\Omega_{C_{n}}\right)_{\Gamma_{C_{n}}}\right)$ is trivial by induction. 


\section{The main construction}

Let $G$ be a finite group which is not cyclic and $r$ be the minimal number of elements required to generate $G$. Since $G$ is not cyclic $r \geq 2$. Pick distinct $f(1), \ldots, f(r) \in$ $G \backslash\{1\}$ such that $G=\langle f(i) \mid 1 \leq i \leq r\rangle$. Set $f(0)=f(1)$.

\subsection{Definitions}

In order to define a fence $\Gamma_{G}$ with stabiliser $G$ we require a $G$-space. Let $G_{0}$, $G_{1}, \ldots, G_{r}, \widehat{G_{0}}, \widehat{G_{1}}, \ldots, \widehat{G_{r}}$ be disjoint copies of $G$ and set

$$
\Omega_{G}=\bigcup_{i=0}^{r}\left(G_{i} \cup \widehat{G_{i}}\right) .
$$

We will write $g_{i}$ (respectively $\widehat{g_{i}}$ ) for the element of $G_{i}$ (respectively $\widehat{G_{i}}$ ) corresponding to $g \in G$. The group $G$ acts on $\Omega_{G}$ by right multiplication:

$$
\begin{aligned}
& g_{i} h=(g h)_{i} \\
& \widehat{g_{i}} h=\widehat{(g h)_{i}} \quad \text { for } g, h \in G \text { and } i \in[0, r]
\end{aligned}
$$

For each $i \in[0, r]$ we define

$$
\begin{array}{rlrl}
\mathcal{R}_{i} & =R_{i} G & \text { with } & R_{i}=\left\{1_{i}, \widehat{1_{i}}, 1_{i+1}, \widehat{1_{i+1}}\right\} \\
\mathcal{T}_{i}=T_{i} G & \text { with } & T_{i}=\left\{\widehat{1_{i}}, 1_{i+1}, \widehat{1_{i+1}}, f(i)_{i+2}\right\}
\end{array}
$$

where all addition is modulo $(r+1)$. We define

$$
\Gamma_{G}=\bigcup_{i=0}^{r}\left(\mathcal{R}_{i} \cup \mathcal{T}_{i}\right) .
$$

and observe that $\Gamma_{G}$ is a $\left(4, \Omega_{G}\right)$-fence and a union of $G$-orbits, so $G \leq \operatorname{Sym}\left(\Omega_{G}\right)_{\Gamma_{G}}$.

\subsection{Intersections}

The proofs in the next section rely on the following detailed analysis of intersections between members of $\Gamma_{G}$.

Lemma 3.1. Let $g \in G$ and $i \in[0, r]$. If $U \in \Gamma_{G}$ then

$$
\left|R_{i} g \cap U\right|= \begin{cases}4 & \text { if } U=R_{i} g \\ 3 & \text { if } U=T_{i} g \\ 2 & \text { if } U \in\left\{T_{i-1} g, R_{i+1} g, R_{i-1} g\right\} \\ 1 & \text { if } U \in\left\{T_{i-1} f(i-1)^{-1} g, T_{i+1} g, T_{i-2} f(i-2)^{-1} g\right\} \\ 0 & \text { otherwise }\end{cases}
$$




$$
\left|T_{i} g \cap U\right|= \begin{cases}4 & \text { if } U=T_{i} g \\ 3 & \text { if } U=R_{i} g \\ 2 & \text { if } U=R_{i+1} g \\ 1 & \text { if } U \in\left\{R_{i+1} f(i) g, T_{i-1} g, T_{i-1} f(i-1)^{-1} g,\right. \\ & \left.\quad T_{i+1} g, T_{i+1} f(i) g, R_{i-1} g, R_{i+2} f(i) g\right\} \\ 0 & \text { otherwise }\end{cases}
$$

Proof: Each $\omega \in \Omega_{G}$ is contained in exactly 4 members of $\Gamma_{G}$ and $|U|=4$ so $\sum_{V \in \Gamma_{G}}|U \cap V|=16$. Hence if there exists a positive integer $s$ and distinct $V_{1}, \ldots, V_{s} \in \Gamma_{G}$ such that $\sum_{i=1}^{s}\left|U \cap V_{i}\right|=16$ then $U \cap V=\emptyset$ for all $V \in \Gamma_{G} \backslash$ $\left\{V_{1}, \ldots, V_{s}\right\}$.

Corollary 3.2. Let $g \in G$ and $i \in[0, r]$. If $h \in \operatorname{Sym}\left(\Omega_{G}\right)_{\Gamma_{G}}$ then there exist $g^{\prime} \in G$ and an integer $i^{\prime}$ such that $0 \leq i^{\prime} \leq r$ and $\left(R_{i} g\right) h=R_{i^{\prime}} g^{\prime}$.

Proof: By Lemma 3.1, if $k \in G$ and $j \in[0, r]$ then exactly three members of $\Gamma_{G}$ intersect $R_{j} k$ in two points whereas only one member of $\Gamma_{G}$ intersects $T_{j} k$ in two points.

Corollary 3.3. Let $g, g^{\prime} \in G$ and $i, i^{\prime} \in[0, r]$.

1. If $h \in \operatorname{Sym}\left(\Omega_{G}\right)_{\Gamma_{G}}$ such that $\left(R_{i} g\right) h=R_{i^{\prime}} g^{\prime}$ then $\left(T_{i} g\right) h=T_{i^{\prime}} g^{\prime}$.

2. If $h \in \operatorname{Sym}\left(\Omega_{G}\right)_{\Gamma_{G}}$ such that $\left(T_{i} g\right) h=T_{i^{\prime}} g^{\prime}$ then $\left(R_{i+1} g\right) h=R_{i^{\prime}+1} g^{\prime}$.

Proof: By Lemma 3.1, if $k \in G$ and $j \in[0, r]$ then $T_{j} k$ is the unique member of $\Gamma_{G}$ which shares exactly three points with $R_{j} k$ and $R_{j+1} k$ is the unique member of $\Gamma_{G}$ which shares two points with $T_{j} k$.

\subsection{The proof}

We set $\mathcal{S}=\left\{R_{i}, T_{i} \mid 0 \leq i \leq r\right\}$ and observe that $\mathcal{S} G$ is a partition of $\Gamma_{G}$ into $|G|$ sets of size $2(r+1)$.

Lemma 3.4. Let $g \in G$. If $h \in \operatorname{Sym}\left(\Omega_{G}\right)_{\Gamma_{G}}$ then there exist an integer $j$ and $g^{\prime} \in G$ such that $\left(R_{i} g\right) h=R_{i+j} g^{\prime}$ and $\left(T_{i} g\right) h=T_{i+j} g^{\prime}$ for all integers $i \in[0, r]$. In particular, if $h \in \operatorname{Sym}\left(\Omega_{G}\right)_{\Gamma_{G}}$ then there exists $g^{\prime} \in G$ such that $(\mathcal{S} g) h=\mathcal{S} g^{\prime}$.

Proof: Corollary 3.2 shows that there exists an integer $j$ and $g^{\prime} \in G$ such that $\left(R_{0} g\right) h=R_{j} g^{\prime}$. The rest follows by induction using Corollary 3.3.

Lemma 3.5. Let $g \in G$. If $h \in \operatorname{Sym}\left(\Omega_{G}\right)_{\Gamma_{G}}$ such that $(\mathcal{S} g) h=\mathcal{S} g$ then

1. $\left(R_{i} g\right) h=R_{i} g$;

2. $\left(T_{i} g\right) h=T_{i} g$;

3. $(\mathcal{S}(f(i) g)) h=\mathcal{S}(f(i) g)$

for all $i \in[0, r]$. 
Proof: By Lemma 3.4 there exists an integer $j$ such that $\left(R_{i} g\right) h=R_{i+j} g$ and $\left(T_{i} g\right) h=T_{i+j} g$ for all integers $i \in[0, r]$. The element $(f(s) g)_{s+2}$ is the unique member of $T_{s} g \backslash R_{s} g$ for all $s \in[0, r]$ so $(f(i) g)_{i+2} h=(f(i+j) g)_{i+j+2}$ for all $i$ such that $0 \leq i \leq r$. Given $s$ such that $0 \leq s \leq r$ the three members of $\Gamma_{G} \backslash\left\{T_{s} g\right\}$ which contain $(f(s) g)_{s+2}$ are all contained in $\mathcal{S}(f(s) g)$ hence $(\mathcal{S}(f(i) g)) h=\mathcal{S}(f(i+j) g)$ by Lemma 3.4. So

$$
\mathcal{S}(f(j) g)=(\mathcal{S}(f(0) g)) h=(\mathcal{S}(f(1) g)) h=\mathcal{S}(f(j+1) g),
$$

therefore $j=0$.

Lemma 3.6. Let $g \in G$ and $i \in[0, r]$. The element $g_{i}$ is the unique member of $R_{i} g \cap$ $T_{i-2} f(i-2)^{-1} g$ and $\widehat{g_{i}}$ is the unique member of $T_{i-1} g \cap T_{i} g$.

Theorem 3.7. $\operatorname{Sym}\left(\Omega_{G}\right)_{\Gamma_{G}} \cong G$.

Proof: By Lemma 3.4 the orbit of $\mathcal{S}$ under $\operatorname{Sym}\left(\Omega_{G}\right)_{\Gamma_{G}}$ is the same as the orbit under $G$. Hence

$$
\left|\operatorname{Sym}\left(\Omega_{G}\right)_{\Gamma_{G}}\right|=|G| \cdot\left|\left(\operatorname{Sym}\left(\Omega_{G}\right)_{\Gamma_{G}}\right)_{\mathcal{S}}\right|
$$

and since $G \leq \operatorname{Sym}\left(\Omega_{G}\right)_{\Gamma_{G}}$ it is enough to show that $\left(\operatorname{Sym}\left(\Omega_{G}\right)_{\Gamma_{G}}\right)_{\mathcal{S}}=1$.

Let $h \in \operatorname{Sym}\left(\Omega_{G}\right)_{\Gamma_{G}}$ such that $\mathcal{S} h=\mathcal{S}$. For $g \in G$ we will write $|g|$ for the length of the shortest word in $\{f(1), \ldots, f(r)\}$ which is equal to $g$. Induction on $|g|$ using Lemma 3.5 shows that $(\mathcal{S} g) h=\mathcal{S} g$ for all $g \in G$. Hence $U h=U$ for all $U \in \Gamma_{G}$ by Lemma 3.5. So $\omega h=\omega$ for all $\omega \in \Omega_{G}$ since there exists $V, W \in \Gamma_{G}$ such that $V \cap W=\{\omega\}$ by Lemma 3.6.

Theorem 3.7 completes the proof of Theorem 1.2.

Remark 3.8. The stabilser of the partitions $\alpha_{\Gamma_{G}}, \beta_{\Gamma_{G}} \in \Omega_{k, l}$ constructed from $\Gamma_{G}$ by Lemma 1.5 can be shown to lie in $A_{k l}$. Hence $\left(A_{k l}\right)_{\alpha_{\Gamma_{G}}, \beta_{\Gamma_{G}}} \cong G$. See [2] for details.

Acknowledgment The author would like to thank the Engineering and Physical Sciences Research Council for providing financial support. He would also like to thank his supervisor Professor Jan Saxl and a referee for their comments which have substantially improved this paper.

\section{References}

1. J.P. James, "Partition actions of symmetric groups and regular bipartite graphs," Bulletin of the London Mathematical Society 38 (2006), 224-232.

2. J.P. James, PhD thesis, The University of Cambridge, (2006). In preparation.

3. W.M. Kantor, "Automorphisms and isomorphisms of symmetric and affine designs," Journal of Algebraic Combinatorics 3 (1994), 307-338. 